\title{
BMJ Open Antiretroviral drug class and anaemia risk in the current treatment era among people living with HIV in the USA: a clinical cohort study
}

\author{
Barbara N Harding (D) , ${ }^{1}$ Bridget M Whitney, ${ }^{1}$ Robin M Nance, ${ }^{1}$ Heidi M Crane,${ }^{2}$ \\ Greer Burkholder, ${ }^{3}$ Richard D Moore, ${ }^{4}$ W Christopher Mathews, ${ }^{5}$ Joseph J Eron, ${ }^{6}$ \\ Peter W Hunt, ${ }^{7}$ Paul Volberding, ${ }^{8}$ Benigno Rodriguez, ${ }^{9}$ Kenneth Mayer, ${ }^{10}$ \\ Michael S Saag, ${ }^{3}$ Mari M Kitahata, ${ }^{2}$ Susan R Heckbert, ${ }^{1}$ Joseph A C Delaney ${ }^{1}$
}

To cite: Harding BN,

Whitney BM, Nance RM, et al. Antiretroviral drug class and anaemia risk in the current treatment era among people living with HIV in the USA: a clinical cohort study. BMJ Open 2020;10:e031487. doi:10.1136/ bmjopen-2019-031487

- Prepublication history and additional material for this paper are available online. To view these files, please visit the journal online (http://dx.doi. org/10.1136/bmjopen-2019031487).

Received 08 May 2019

Revised 17 February 2020

Accepted 25 February 2020

Check for updates

(C) Author(s) (or their employer(s)) 2020. Re-use permitted under CC BY-NC. No commercial re-use. See rights and permissions. Published by BMJ.

For numbered affiliations see end of article.

Correspondence to Dr Barbara N Harding; hardingb@uw.edu

\section{ABSTRACT}

Objective Anaemia is common among people living with HIV (PLWH) and has been associated with certain, often older, antiretroviral medications. Information on current antiretroviral therapy (ART) and anaemia is limited. The objective was to compare the associations between anaemia incidence or haemoglobin change with core ART classes in the current ART era.

Design Retrospective cohort study.

Setting USA-based prospective clinical cohort of PLWH aged 18 and above receiving care at eight sites between January 2010 and March 2018.

Participants 16505 PLWH were included in this study. Main outcome measures Anaemia risk and haemoglobin change were estimated among PLWH for person-time on a protease inhibitor $(\mathrm{PI})$ or an integrase strand transfer inhibitor (INSTI)-based regimen, relative to a non-nucleoside reverse transcriptase inhibitor (NNRTI)based reference. We also examined PLWH on regimens containing multiple core classes. Cox proportional hazards regression analyses were conducted to measure the associations between time-updated ART classes and incident anaemia or severe anaemia. Linear mixed effects models were used to examine the relationships between ART classes and haemoglobin change.

Results During a median of 4.9 years of follow-up, 1040 developed anaemia and 488 developed severe anaemia. Compared with NNRTI use, INSTI-based regimens were associated with an increased risk of anaemia (adjusted HR (aHR) $1.26,95 \% \mathrm{Cl} 1.00$ to 1.58 ) and severe anaemia (aHR $1.51,95 \% \mathrm{Cl} 1.07$ to 2.11 ) and a decrease in haemoglobin level. Time on multiple core classes was also associated with increased anaemia risk (aHR $1.39,95 \% \mathrm{Cl} 1.13$ to 1.70), while no associations were found for PI use. Conclusion These findings suggest INSTI use may increase the risk of anaemia. If confirmed, screening for anaemia development in users of INSTIs may be beneficial. Further research into the underlying mechanisms is warranted.

\section{INTRODUCTION}

Anaemia and severe anaemia are common among people living with HIV (PLWH). ${ }^{1}$ The

\section{Strengths and limitations of this study}

- This study used a large and geographically diverse population of people living with HIV in care across the USA

- This study leveraged comprehensive clinical data, including information on diagnoses, medication use, laboratory test results, demographic information and medical history.

- This study investigated the associations between specific types of antiretroviral therapy core regimens and anaemia risk.

- This observational study is subject to residual confounding.

- This study focused on anaemia assessed from haemoglobin lab values taken at regular medical care visits without excluding participants with conditions strongly associated with haemoglobin level through mechanisms unrelated to HIV infection.

prevalence of anaemia is elevated in PLWH compared with the general population. One study reported that among non-pregnant American women living with HIV, the prevalence of anaemia was $28.1 \%$ compared with $15.1 \%$ among women without HIV. ${ }^{2}$ Estimates vary by age, sex, HIV disease stage, use of antiretroviral therapy (ART) and injection drug use status. ${ }^{13}$ Among PLWH, associations have been found between anaemia and mortality, ${ }^{4-9}$ health-related quality of life, ${ }^{1}$ morbidity, dementia ${ }^{10}$ and ART failure. ${ }^{11}$ In addition, anaemia is an independent prognostic indicator associated with HIV disease progression, ${ }^{1} 1213$ including development of AIDS. $^{7}$

Research shows that ART impacts anaemia risk among PLWH. In the early treatment era, use of zidovudine (AZT) was a cause of bone marrow suppression leading to anaemia. ${ }^{14}$ 


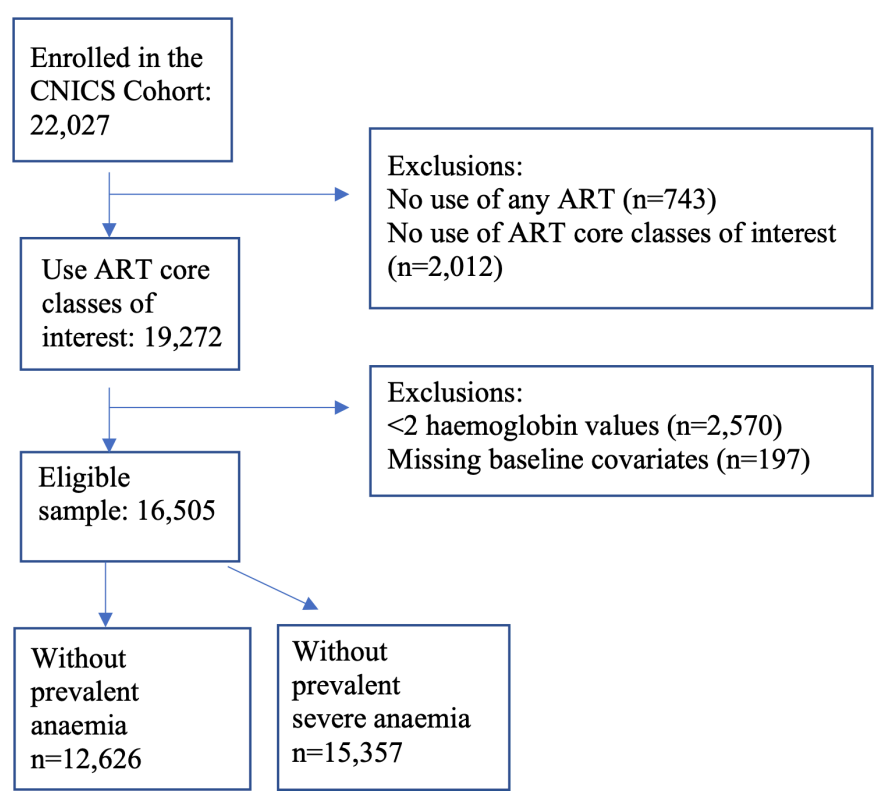

Figure 1 Flow chart of inclusion/exclusion criteria for 22027 PLWH in care at CNICS after January 2010. Exclusions were made for those not exposed to any of the ART core classes, those with fewer than two haemoglobin levels and those missing baseline covariates, resulting in $16505 \mathrm{PLWH}$ who were included in these analyses. ART, antiretroviral therapy; CNICS, Centers for AIDS Research Network of Integrated Clinical Systems; PLWH, people living with HIV.

However, in recent years, AZT use has decreased substantially as other, better tolerated ART medications have become available. Despite the impact of specific agents such as AZT, ART use in general is associated with reduced anaemia incidence, ${ }^{15} 16$ likely due to inhibition of HIV disease progression. ${ }^{17}$ Current ART regimens typically include a pair of nucleoside reverse transcriptase inhibitors (NRTIs) as a backbone plus a core agent. Common core classes include non-nucleoside reverse transcriptase inhibitors (NNRTIs), integrase strand transfer inhibitors (INSTIs) and protease inhibitors (PIs). While ART use overall reduces anaemia, little is known about whether anaemia risk differs between commonly used ART classes in the current treatment era, particularly the newer INSTI class. From clinical safety data of trials, 36\%-49\% of participants using PIs had haemoglobin $(\mathrm{Hb})$ levels $<10 \mathrm{~g} / \mathrm{dL}$, indicating anaemia, ${ }^{18}$ and in a randomised controlled trial two participants discontinued INSTI use due to anaemia adverse events. ${ }^{19}$ However, many studies included few participants or were mostly from an earlier ART era when older ART medications were predominantly used or from trials that may be less generalisable to the diverse population of PLWH in clinical care. The objective of this study was to compare the rates of anaemia and severe anaemia development as well as changes in $\mathrm{Hb}$ over time based on classes of ART used in the current treatment era.

\section{METHODS}

\section{Overview and setting}

The present study included PLWH in care in the Centers for AIDS Research Network of Integrated Clinical Systems (CNICS) cohort during the period from 1 January 2010 to 31 March 2018 (the date through which each site had complete data (administrative censor date) varied somewhat, median date: 31 October 2017). The CNICS cohort has been described in detail elsewhere. ${ }^{20}$ Briefly, CNICS is a dynamic prospective clinical cohort of >32000 adult PLWH receiving care at eight participating sites across the USA. Comprehensive clinical data, including diagnoses, ART and other medications, laboratory test results, demographic information, and historical information, including ART use before enrolment, are collected through electronic medical records and other institutional data systems at each site and harmonised in the CNICS data repository. Medication data including ART use are entered into the electronic medical records by clinicians, or prescription fill/refill data are uploaded directly from pharmacy systems and verified through medical record review. Participants entered the current study on 1 January 2010 or the earliest date after 1 January 2010 that they met the following enrolment criteria (cohort entry date): (1) enrolment in CNICS for $\geq 6$ months to allow time for covariate ascertainment and (2) use of an ART regimen containing a backbone of two NRTIs plus a PI, NNRTI or INSTI. In addition, all participants were required to have at least two available $\mathrm{Hb}$ lab values during the study follow-up. Figure 1 shows the inclusion criteria and the exclusions made. Informed consent was obtained from all participants.

\section{Exposure}

The exposure of interest was the ART core drug class (NNRTI, INSTI or PI) prescribed as part of an ART regimen (a backbone of two NRTIs plus a core drug). Participants switching to different core drugs within the same class were considered to be continually exposed to the same core drug class. Individuals with a gap of 6 or more months in use of the ART core drug classes of interest were censored at the start of the gap and did not re-enter the study.

Person-time on INSTI or PI-based regimens was compared with the NNRTI reference. In addition, some PLWH in this cohort had prescriptions for multiple core classes simultaneously. Participants with regimens containing more than one core class were categorised separately in analyses as users of 'multiple core classes'. Boosting agents (eg, boosted ritonavir or cobicistat) were not considered a second core agent.

\section{Outcome ascertainment}

$\mathrm{Hb}$ levels, expressed in grams per decilitre, were ascertained using inpatient and outpatient laboratory data obtained as part of clinical care. Outcomes included incident anaemia (first postbaseline $\mathrm{Hb}$ measure below $10 \mathrm{~g} / \mathrm{dL}$ ), incident severe anaemia (first postbaseline 
$\mathrm{Hb}$ measure below $7.5 \mathrm{~g} / \mathrm{dL})^{21}$ and changes in $\mathrm{Hb}$ level. Another outcome, chronic anaemia, defined as anaemia lasting for $\geq 6$ months, was also examined. Chronic anaemia was defined as postbaseline $\mathrm{Hb}$ lab results on two separate occasions at least 6 months apart which were consistently in the anaemic range without any $\mathrm{Hb}$ values above the anaemia range during this 6 -month period.

\section{Participant characteristics}

Characteristics that were analysed as confounders of the association between ART core drugs and incident anaemia, severe anaemia or change in $\mathrm{Hb}$ over time included age, sex, race/ethnicity, CNICS site, hepatitis $\mathrm{C}$ virus (HCV) coinfection defined as a detectable HCV RNA level or HCV genotype or HCV antibody, kidney function measured using estimated glomerular filtration rate (eGFR; categorised as $<30,30-59$ or $\geq 60 \mathrm{~mL}$ / $\mathrm{min} / 1.73 \mathrm{~m}^{2}$ ), ${ }^{22} \mathrm{CD} 4$ count (categorised as $\geq 500$, $350-499,200-399,100-199$ or $<100$ cells $/ \mathrm{mm}^{3}$ ), viral load (VL, assessed as $\log _{10}(\mathrm{VL}+1)$ ), baseline $\mathrm{Hb}$ (in incident anaemia, severe anaemia and chronic anaemia analyses only), and time in care at CNICS sites, defined as time from cohort entry date until the last available CNICS activity: either last lab date or last visit. HCV, eGFR, CD4 count and VL were assessed as part of clinical care visits and were time-updated as repeated measures occurred. All covariates were selected a priori, based on review of the literature and clinical knowledge. In addition, assessment of self-reported ART adherence was available for a subset of $\sim 55 \%$ of the study population who were in care after each individual site initiated a clinical assessment of patient-reported outcomes, including adherence. ${ }^{23}$

\section{Statistical analysis}

Baseline characteristics are presented for all participants at the cohort entry date. Median and IQR are displayed for continuous variables, and frequencies and proportions are displayed for categorical variables.

Two multivariable Cox proportional hazards regression analyses were conducted, one among the subset of PLWH who were anaemia-free at baseline to determine associations between time-updated NNRTI, PI and INSTI use and development of anaemia, and another among the subset of participants who were free of severe anaemia at baseline to determine associations between time-updated NNRTI, PI and INSTI use and development of severe anaemia.

Participants were censored at (1) the time they developed the outcome of interest, (2) at the time of last activity in CNICS, (3) at the time of death, (4) at the date of administrative censoring at each site or (5) at the time they no longer were prescribed one of the ART core classes of interest, whichever came first. The timescale for the models was time since cohort entry. Complete case analysis methods were used ( $<2 \%$ had missing data).

In a sensitivity analysis, we examined those who were ART-naïve at baseline and who initiated a regimen, including one of the core ART classes of interest during study follow-up. Follow-up in this analysis began when a person began their initial ART regimen and extended until the earliest time of anaemia occurrence, last activity in CNICS, time of death, administrative censoring or at the time their initial regimen ended. PI or INSTI use was compared with the reference, NNRTI use. We also examined the change in $\mathrm{Hb}$ over time using mixed models among this ART-naïve population. We also conducted sensitivity analyses including time-updated NRTI backbone regimen adjustment in analyses of incident anaemia and incident severe anaemia risk. These sensitivity analyses addressed possible concerns that the NRTI backbone may influence anaemia risk rather than the core agent. Finally, we conducted a sensitivity analysis that excluded users of AZT from comparisons of NNRTI, PI and INSTI use versus the referent category of NNRTI use because of concerns that AZT has been found strongly associated with anaemia.

Linear mixed effects models with random slopes for time were used to examine the association of ART core classes with Hb levels among all PLWH after adjustment for the same characteristics as in the incident anaemia and severe anaemia analyses. Mixed effects models use random slopes and intercepts at the participant level to handle irregular patterns of repeated measures over follow-up. ${ }^{24}$ All analyses were performed using Stata V.14.2.

\section{Patient and public involvement}

There was no patient or public participation in the present study.

\section{RESULTS}

In total, 16505 PLWH met the inclusion criteria and were included in these analyses (figure 1). Participants had an average of 11 outpatient $\mathrm{Hb}$ values measured during a median follow-up of 4.9 (IQR 3.0-7.2) years. A total of $12626(76 \%)$ were free of anaemia at baseline, and 15357 $(93 \%)$ were free of severe anaemia at baseline. Table 1 provides the baseline characteristics of the study participants in the analyses of incident anaemia and incident severe anaemia. Overall, the mean age of study participants was 46 years at cohort entry, $20 \%$ were female and $19 \%$ were coinfected with $\mathrm{HCV}$. At baseline, $18 \%$ were prescribed regimens with an NNRTI, 53\% with a PI, $14 \%$ an INSTI and $16 \%$ regimens with multiple cores. INSTIs were increasingly used over the last few years of the study period (figure 2), and among those simultaneously prescribed multiple core medications the proportion comprising INSTI plus another core class increased as study years progressed. In the analytic study sample, backbone regimens mainly consisted of emtricitabine/ lamivudine (3TC) plus tenofovir or 3TC plus abacavir (online supplementary table 1 ).

The overall incidence of anaemia was 2.1 per 100 personyears, and the overall incidence of severe anaemia was 0.8 per 100 person-years. The unadjusted incidence rates of 
Table 1 Baseline characteristics of PLWH in CNICS who were receiving an ART core agent of interest $(n=16505)^{\star}$

\begin{tabular}{|c|c|c|c|c|}
\hline & \multicolumn{2}{|c|}{ Incident anaemia analysis $(\mathrm{n}=12626)$} & \multicolumn{2}{|c|}{ Incident severe anaemia analysis $(n=15357)$} \\
\hline & $\begin{array}{l}\text { Do not develop } \\
\text { anaemia }(n=11586)\end{array}$ & $\begin{array}{l}\text { Develop anaemia } \\
(n=1040)\end{array}$ & $\begin{array}{l}\text { Do not develop severe } \\
\text { anaemia }(n=14869)\end{array}$ & $\begin{array}{l}\text { Develop severe } \\
\text { anaemia }(n=488)\end{array}$ \\
\hline Age, median (IQR) & $45(37-51)$ & $47(40-54)$ & $45(37-52)$ & $46(39-54)$ \\
\hline Female & $1574(14)$ & $276(27)$ & $2681(18)$ & $158(32)$ \\
\hline \multicolumn{5}{|l|}{ Race/ethnicity } \\
\hline White & $5782(50)$ & $396(38)$ & $6840(46)$ & $157(32)$ \\
\hline Black & $3720(32)$ & $499(48)$ & $5442(37)$ & $254(52)$ \\
\hline Hispanic & $1537(13)$ & $106(10)$ & $1920(13)$ & $58(12)$ \\
\hline Other/missing & $547(5)$ & $39(4)$ & $667(4)$ & $19(4)$ \\
\hline $\begin{array}{l}\text { Years in CNICS at cohort entry*, } \\
\text { median (IQR) }\end{array}$ & $5.2(2.3-8.8)$ & $5.8(2.4-9.5)$ & $5.5(2.4-9.0)$ & $5.5(2.8-9.1)$ \\
\hline Viral load $\geq 400$ copies $/ \mathrm{mL}$ & $2441(21)$ & $283(27)$ & 3259 (22) & $172(35)$ \\
\hline \multicolumn{5}{|l|}{ CD4 count (cells $\left./ \mathrm{mm}^{3}\right)$} \\
\hline$<100$ & $528(5)$ & $112(11)$ & $915(6)$ & $90(19)$ \\
\hline $100-199$ & $870(8)$ & $96(9)$ & $1256(8)$ & $63(13)$ \\
\hline 200-349 & $1974(17)$ & $216(21)$ & 2675 (18) & $113(23)$ \\
\hline $350-499$ & $2497(21)$ & $226(22)$ & $3160(21)$ & $80(16)$ \\
\hline$\geq 500$ & $5717(49)$ & $390(38)$ & $6863(46)$ & $142(29)$ \\
\hline Hepatitis $\mathrm{C}$ virus coinfection & $1816(16)$ & $303(29)$ & $2711(18)$ & $139(28)$ \\
\hline \multicolumn{5}{|c|}{ Kidney function (eGFR) $\left(\mathrm{mL} / \mathrm{min} / 1.73 \mathrm{~m}^{2}\right)$} \\
\hline$<30$ & $32(<1)$ & $36(3)$ & $142(1)$ & $42(9)$ \\
\hline $30-59$ & $459(4)$ & $80(8)$ & $731(5)$ & $51(10)$ \\
\hline$\geq 60$ & $11095(96)$ & $924(89)$ & $13996(94)$ & $395(81)$ \\
\hline $\begin{array}{l}\text { Baseline haemoglobin ( } \mathrm{g} / \mathrm{dL} \text { ), } \\
\text { median (IQR) }\end{array}$ & $14.5(13.5-15.4)$ & $13.3(12.2-14.4)$ & $14.3(13.1-15.2)$ & $12.4(10.8-13.8)$ \\
\hline \multicolumn{5}{|l|}{$\mathrm{BMI}\left(\mathrm{kg} / \mathrm{m}^{2}\right)$} \\
\hline$<18.5$ & $229(2)$ & $36(3)$ & $377(3)$ & $30(6)$ \\
\hline 18.5 to $<25.0$ & $4806(41)$ & $426(41)$ & $6120(41)$ & $211(43)$ \\
\hline 25.0 to $<30.0$ & 3929 (34) & $301(29)$ & $4885(33)$ & $117(24)$ \\
\hline$\geq 30.0$ & $2622(23)$ & $277(27)$ & $3487(23)$ & $130(27)$ \\
\hline \multicolumn{5}{|l|}{ ART core class } \\
\hline NNRTI & $2109(18)$ & $177(17)$ & $2633(18)$ & $70(14)$ \\
\hline $\mathrm{PI}$ & $6135(53)$ & $558(54)$ & 7935 (53) & $251(51)$ \\
\hline INSTI & $1803(16)$ & $93(9)$ & $2126(14)$ & $46(9)$ \\
\hline Multiple core classes & $1539(13)$ & $212(20)$ & $2175(15)$ & $121(25)$ \\
\hline $\begin{array}{l}\text { Self-reported adherence (on a } \\
100-\text { point scale), median (IQR) }\end{array}$ & $98(93-100)$ & 98 (91-99) & $98(92-100)$ & 97 (90-99) \\
\hline
\end{tabular}

${ }^{*}$ Cohort entry date was defined as the earliest date during 1 January 2010-31 March 2018 that a person had $\geq 6$ months in CNICS and was receiving an ART regimen with a core agent of interest.

†For the $55 \%$ of the population who reported medication adherence.

ART, antiretroviral therapy; BMI, body mass index; CNICS, Centers for AIDS Research Network of Integrated Clinical Systems; eGFR, estimated glomerular filtration rate; INSTI, integrase strand transfer inhibitor; NNRTI, non-nucleoside reverse transcriptase inhibitor; PI, protease inhibitor; PLWH, people living with HIV.

anaemia and severe anaemia based on ART core class are provided in table 2. In adjusted analyses, INSTI use was associated with an increased risk of anaemia (adjusted HR (aHR) $1.26,95 \%$ CI 1.00 to 1.58 ) compared with NNRTIs (table 3). Use of multiple core classes together was also associated with an increased risk of anaemia (aHR 1.39, 95\% CI 1.13 to 1.70 ), while no associations were found between PI use and anaemia (aHR of 1.09, 95\% CI 0.90 to 


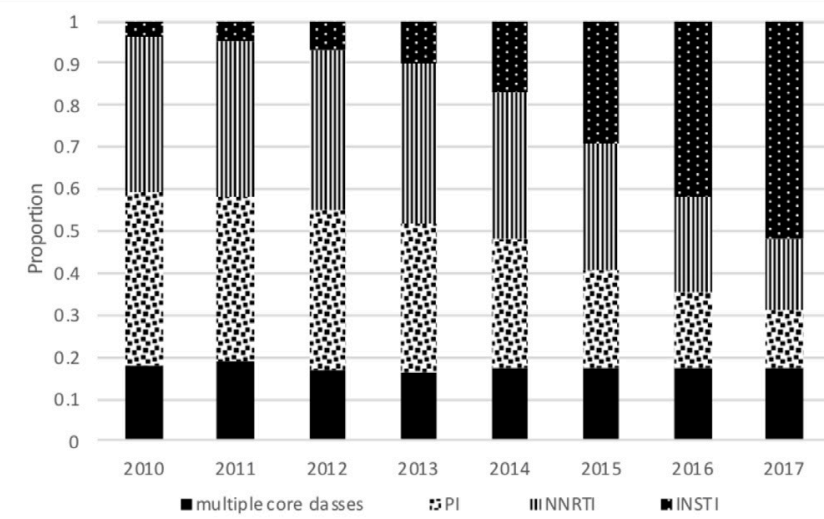

Figure 2 Proportion of study population ( $n=16505)$ using various ART classes during complete years of study followup. This figure shows the trends in use of the ART core classes during 2010-2017. ART, antiretroviral therapy; INSTI, integrase strand transfer inhibitor; NNRTI, non-nucleoside reverse transcriptase inhibitor; $\mathrm{PI}$, protease inhibitor.

1.32). In adjusted analyses restricted to participants free of severe anaemia at baseline (table 3), INSTI use was associated with an increased risk of severe anaemia (aHR 1.51, 95\% CI 1.07 to 2.11) compared with NNRTI use. Although the HR appeared elevated, there was no association between time on multiple ART core classes and an increased risk of severe anaemia (aHR 1.30, 95\% CI 0.95 to 1.78 ), and no association was found for PIs (aHR $1.09,95 \%$ CI 0.81 to 1.47 ). Among the 12626 PLWH who were free of anaemia at baseline, 225 developed chronic anaemia (lasting for $\geq 6$ months) during follow-up. For

Table 2 Incidence rate of anaemia (haemoglobin $<10 \mathrm{~g} / \mathrm{dL}$ ) and severe anaemia (haemoglobin $<7.5 \mathrm{~g} / \mathrm{dL}$ ) by ART core drug class

\begin{tabular}{lccl}
\hline $\begin{array}{l}\text { ART } \\
\text { regimen }\end{array}$ & $\begin{array}{c}\text { Follow-up time } \\
\text { (person-years) }\end{array}$ & Events & $\begin{array}{l}\text { Rate (per 100 } \\
\text { person-years) }\end{array}$ \\
\hline Anaemia & & 150 & 1.50 \\
\hline NNRTI & 9964 & 485 & 1.96 \\
\hline PI & 24710 & 155 & 2.10 \\
INSTI & 7389 & 250 & 3.06 \\
Multiple & 8172 & & \\
$\begin{array}{l}\text { core } \\
\text { classes }\end{array}$ & & & \\
$\begin{array}{l}\text { Severe } \\
\text { anaemia }\end{array}$ & & 57 & 0.47 \\
\hline NNRTI & 12113 & 204 & 0.65 \\
\hline $\begin{array}{l}\text { PI } \\
\text { INSTI }\end{array}$ & 31156 & 84 & 0.92 \\
\hline $\begin{array}{l}\text { Multiple } \\
\text { core } \\
\text { classes }\end{array}$ & 11258 & 143 & 1.27 \\
\hline
\end{tabular}

ART, antiretroviral therapy; INSTI, integrase strand transfer inhibitor; NNRTI, non-nucleoside reverse transcriptase inhibitor; PI, protease inhibitor.

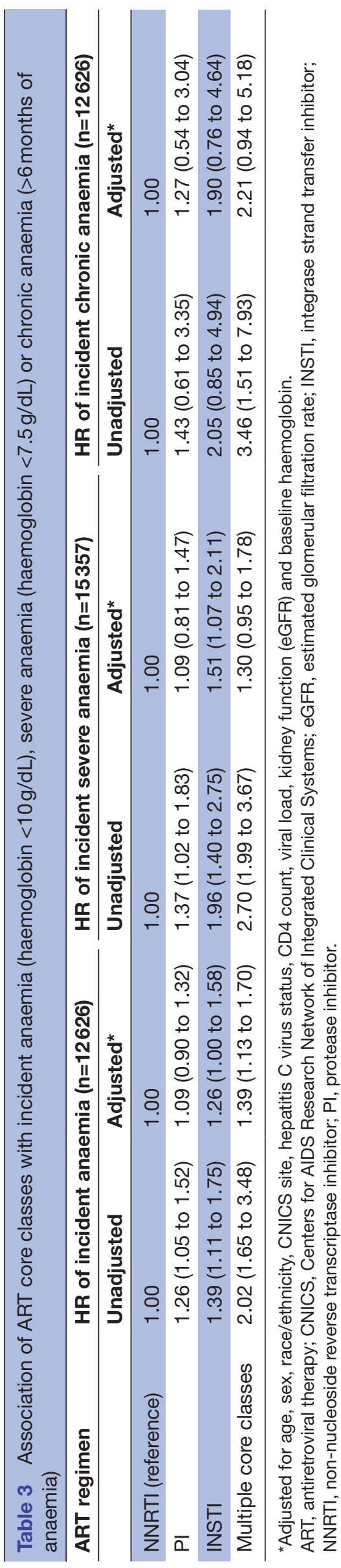


Table 4 Association of ART core classes with change in haemoglobin level during follow-up in adjusted analyses (linear mixed effect model) $(n=16505)$

\begin{tabular}{lllr}
\hline ART class & Coefficient* $^{*}$ & $\mathbf{9 5 \%} \mathbf{C l}$ & P value \\
\hline NNRTI (reference) & & & \\
PI & -0.01 & -0.04 to 0.03 & 0.675 \\
INSTI & -0.06 & -0.10 to -0.03 & $<0.001$ \\
$\begin{array}{l}\text { Multiple core } \\
\text { classes }\end{array}$ & -0.14 & -0.18 to -0.11 & $<0.001$ \\
\hline
\end{tabular}

${ }^{*}$ Coefficient is the mean difference per year in haemoglobin $(\mathrm{g} /$ $\mathrm{dL})$ for each core regimen relative to the NNRTI core regimen, after adjustment for site, age, sex, race/ethnicity, hepatitis $C$ virus coinfection, CD4 cell count, viral load, eGFR and years in study. ART, antiretroviral therapy; eGFR, estimated glomerular filtration rate; INSTI, integrase strand transfer inhibitor; NNRTI, nonnucleoside reverse transcriptase inhibitor; PI, protease inhibitor.

chronic anaemia, results were similar to those in the primary analysis, although CIs overlapped 1. Relative to NNRTI use, person-time on multiple core classes was associated with an aHR for chronic anaemia of 2.21 (95\% CI 0.94 to 5.18$)$, person-time on an INSTI with an aHR of 1.90 (95\% CI 0.76 to 4.64$)$ and person-time on a PI with an aHR of 1.27 (95\% CI 0.54 to 3.04 ).

Average $\mathrm{Hb}$ levels remained steady during follow-up; the mean level was $14.1 \mathrm{~g} / \mathrm{dL}$ (IQR 12.7-15.1) at baseline and $14.0 \mathrm{~g} / \mathrm{dL}$ (IQR 12.6-15.2) at the last available measurement per person. Relative to NNRTI use, a decrease in $\mathrm{Hb}$ level over time was associated with both INSTI use $(-0.06 \mathrm{~g} / \mathrm{dL}$ per year, $95 \% \mathrm{CI}-0.10$ to 0.03$)$ and use of multiple core classes $(-0.14,95 \%$ CI -0.18 to $0.11)$. No association was found for PI use $(-0.01,95 \% \mathrm{CI}$ -0.04 to 0.03 ) (table 4 ).

The sensitivity analysis restricted to ART-naïve participants included 6426 PLWH who were free of prevalent anaemia at baseline, of whom 378 developed anaemia. Compared with NNRTI initiators, those initiating a PI had an aHR of 0.69 (95\% CI 0.45 to 1.06$)$ while those initiating an INSTI had an aHR of 1.10 (95\% CI 0.84 to 1.44) (online supplementary table 2). The mixed model examining change in Hb over time among ARTnaïve PLWH initiating one of the ART core classes of interest included 7264 participants. Compared with NNRTI initiators, a decrease in $\mathrm{Hb}$ was found for PI use $(-0.08 \mathrm{~g} / \mathrm{dL}$ per year, $95 \% \mathrm{CI}-0.16$ to 0.01$)$, while INSTI use was associated with a larger decrease in $\mathrm{Hb}$ level over time $(-0.15 \mathrm{~g} / \mathrm{dL}$ per year, $95 \% \mathrm{CI}-0.22$ to 0.09) (online supplementary table 3). Results from the sensitivity analyses including time-updated NRTI backbone regimen adjustment were essentially unchanged (online supplementary table 4). Finally, results from the sensitivity analysis excluding participants with AZT use indicated similar findings to those from the primary analyses, although CIs were wider (online supplementary tables 5 and 6).

\section{DISCUSSION}

In this study of 16505 PLWH in care within the USA in the current treatment era (2010 and after), we observed that INSTI use and time on multiple core ART classes were associated with decreases in $\mathrm{Hb}$ levels during follow-up compared with using NNRTI-based regimens. We found that INSTI use was associated with an increased risk of anaemia (aHR 1.26, 95\% CI 1.00 to 1.58) and severe anaemia (aHR 1.51, 95\% CI 1.07 to 2.11) as well as a decrease in $\mathrm{Hb}$ levels over time. Furthermore, the naïve user analysis indicated similar findings despite a smaller sample size. These findings could have implications for the treatment approach that should be used in people with risk factors for anaemia.

This study's strengths include its large and geographically diverse study population and longitudinal data structure. Nevertheless, there are limitations to this study to consider, including the observational nature of the data, which may be subject to residual confounding, including confounding by indication. ${ }^{25}$ However, anaemia is not a recognised adverse effect of NNRTIs, PIs or INSTIs. Thus, it is unlikely that ART core class was selected based on prescriber concern about anaemia risk. Additionally, we did not exclude participants with conditions strongly associated with anaemia or $\mathrm{Hb}$ level, including those on dialysis, receiving erythropoietin or with severe bleeding, which likely caused some of the anaemia cases in this analysis. However, in the sensitivity analysis focusing on factors associated with chronic anaemia (less likely due to bleeding), findings for INSTI versus NNRTI core regimens were similar to those including all PLWH who became anaemic. Information on ART medication use was from prescription data, which does not necessarily indicate medications were taken, although self-reported adherence was high $(\sim 98 \%)$ in the subset for whom adherence information was available. CNICS participants who provided adherence information have been shown to be representative of the overall population of PLWH in CNICS. ${ }^{23}$ Finally, the fact that this study was conducted among PLWH in care in the USA who are on ART may limit the generalisability of findings to PLWH who live outside of the USA.

There have been few epidemiological studies of anaemia risk among users of newer ART core regimens. From clinical safety data reported from multiple trials, approximately $36 \%-49 \%$ of participants using PIs had Hb levels $<10 \mathrm{~g} / \mathrm{dL}$, indicating anaemia, ${ }^{18}$ and in another trial two participants discontinued INSTI use due to anaemia adverse events ${ }^{19}$; however, the strict inclusion criteria applied in clinical trials makes it difficult to generalise these findings to more diverse populations of PLWH in clinical care. Another study, conducted during the newer era of HIV treatment with drugs other than AZT (during 2008-2012), presented findings for AZT versus non-AZT regimens, finding an increased risk of anaemia among AZT compared with non-AZT regimens $(\mathrm{HR}=2.84,95 \% \mathrm{CI} 1.52$ to 5.31$) .{ }^{27}$ However, anaemia risk was not analysed separately for the use of specific classes 
of ART, resulting in the inability of comparison with the present study's findings and a lack of generalisability to PLWH who are treated with newer ART core agents.

It is possible that PLWH in our study whose HIV is progressing due to resistance or other complications may get switched to an INSTI. This, in addition to prior knowledge that poorly controlled HIV parameters are on their own a risk factor for anaemia, ${ }^{5829}$ could result in confounding by indication. However, the switch to INSTI core regimens since their approval in 2007 has been widespread in this population (figure 2) and INSTIs are recommended for use as initial regimens. ${ }^{30}$ In addition, we rigorously controlled for many of the important HIVrelated factors that correspond to poorly controlled HIV, and our sensitivity analysis examining PLWH initiating their initial regimen failed to reinforce the notion that an increased risk of anaemia among INSTI core regimen users could be entirely explained by sicker participants getting switched to these therapies.

PLWH on multiple core classes were in a different category in our analyses. There are several reasons PLWH may be prescribed multiple core classes. For example, sometimes PLWH are prescribed multiple core classes to ensure they receive a complete regimen while awaiting approval for specific agents from their insurance company. However, the primary concern was that they were receiving multiple core classes due to provider concerns such as prior failed regimens, which may also increase their risk of anaemia.

In conclusion, in this large, diverse, multicentre cohort of PLWH, we found that INSTI use and time on multiple ART core classes were associated with progression to anaemia and a lower Hb level. INSTI use was also associated with severe anaemia risk. Our findings suggest that careful selection of ART regimen could mitigate anaemia development, although this anaemia risk needs to be balanced with the possibility of improvement in overall HIV care. ${ }^{31}$ Further research is needed to replicate the finding of INSTI core regimen use and anaemia risk and to understand the underlying mechanisms. If confirmed, screening for anaemia development in users of INSTIs may be beneficial.

\footnotetext{
Author affiliations

${ }^{1}$ Department of Epidemiology, University of Washington, Seattle, Washington, USA

${ }^{2}$ Medicine, University of Washington, Seattle, Washington, USA

${ }^{3}$ University of Alabama at Birmingham, Birmingham, Alabama, USA

${ }^{4}$ Medicine, Johns Hopkins University School of Medicine, Baltimore, Maryland, USA

${ }^{5}$ University of California, San Diego, La Jolla, California, USA

${ }^{6}$ University of North Carolina at Chapel Hill, Chapel Hill, North Carolina, USA

${ }^{7}$ University of California San Francisco, San Francisco, California, USA

${ }^{8}$ Medicine, University of California, San Francisco, San Francisco, California, USA

${ }^{9}$ Case Western Reserve University, Cleveland, Ohio, USA

${ }^{10}$ The Fenway Institute at Fenway Health, Boston, Massachusetts, USA
}

Contributors BNH has conducted all analyses, contributed to methodological approach and wrote the manuscript. In addition, BMW and RMN contributed to data preparation and analysis. JACD contributed to the analysis. HMC, SRH and JACD contributed to conception and design of the work. HMC, GB, RM, WCM, JJE, BR, KM, MSS and MMK contributed to data collection. BNH, BMW, RMN, HMC, GB, RM, WCM,
JJE, PH, PV, BR, KM, MSS, MMK, SRH and JACD contributed to interpretation of data and critically revising the manuscript for important intellectual content.

Funding This project was funded by R01HL126538-01A1/National Heart, Lung, and Blood Institute. They provided an unrestricted grant and we are completely independent from the study sponsors. Additional support came from the National Institute of Allergy and Infectious Diseases (NIAID) at the National Institutes of Health (CNICS R24 AI067039, UW CFAR NIAID grant P30 AI027757, UNC CFAR grant P30 Al50410, JHU CFAR grant P30 Al094189, and UAB CFAR grant P30 Al027767). BNH is supported by a National Heart, Lung, and Blood Institute (grant T32HL007828).

Competing interests BNH reports grants from the National Heart, Lung, and Blood Institute (NHLBI) during the conduct of the study. BMW reports grants from the National Institutes of Health (NIH) during the conduct of the study. RMN reports grants from NIH during the conduct of the study. HMC reports grants from NHLBI during the conduct of the study, grants from $\mathrm{NIH}$, grants from ViiV Healthcare and grants from PCORI outside the submitted work. GB reports grants from NIH during the conduct of the study, and other from Amgen outside the submitted work. RM reports grants from NHLBI during the conduct of the study. WCM reports grants from NHLBI during the conduct of the study. JJE reports grants from NIH during the conduct of the study, grants and personal fees from Gilead Sciences, grants and personal fees from ViiV Healthcare, grants and personal fees from Janssen, and personal fees from Merck outside the submitted work. PH reports grants from NHLBI during the conduct of the study, personal fees from Gilead, personal fees from ViiV Healthcare, personal fees from Janssen and non-financial support from Merck outside the submitted work. PV reports grants from NHLBI during the conduct of the study and other from Merck outside the submitted work. BR reports grants from NIH during the conduct of the study, personal fees from Gilead and personal fees from ViiV outside the submitted work. KM reports grants from NHLBI during the conduct of the study. MSS reports grants from NIAID/NIH during the conduct of the study, and grants from Gilead, Merck and ViiV Healthcare outside the submitted work. MMK reports grants from NHLBI during the conduct of the study. $\mathrm{SRH}$ reports grants from NIH during the conduct of the study. JACD reports grants from NHLBI during the conduct of the study.

Patient and public involvement Patients and/or the public were not involved in the design, or conduct, or reporting, or dissemination plans of this research.

Patient consent for publication Not required.

Ethics approval Institutional review boards at each site approved the CNICS protocols for patient protection and provided general approval for secondary data analysis. The University of Washington Human Subjects Division served as the institutional review board for the centralised deidentified CNICS Data Repository (IRB approval number 27674-D).

Provenance and peer review Not commissioned; externally peer reviewed.

Data availability statement Data may be obtained from a third party and are not publicly available. The Centers for AIDS research (CFAR) Network of Integrated Clinical Systems (CNICS) data may be accessed with an approved concept proposal. Instructions for data access and concept proposal forms may be found at https:// www.uab.edu/ cnics/submit-proposal.

Open access This is an open access article distributed in accordance with the Creative Commons Attribution Non Commercial (CC BY-NC 4.0) license, which permits others to distribute, remix, adapt, build upon this work non-commercially, and license their derivative works on different terms, provided the original work is properly cited, appropriate credit is given, any changes made indicated, and the use is non-commercial. See: http://creativecommons.org/licenses/by-nc/4.0/.

ORCID iD

Barbara N Harding http://orcid.org/0000-0002-9460-9399

\section{REFERENCES}

1 Redig AJ, Berliner N. Pathogenesis and clinical implications of HIVrelated anemia in 2013. Hematology Am Soc Hematol Educ Program 2013;2013:377-81.

2 Semba RD, Shah N, Klein RS, et al. Prevalence and cumulative incidence of and risk factors for anemia in a multicenter cohort study of human immunodeficiency virus-infected and -uninfected women. Clin Infect Dis 2002;34:260-6.

3 Belperio PS, Rhew DC. Prevalence and outcomes of anemia in individuals with human immunodeficiency virus: a systematic review of the literature. Am J Med 2004;116 (Suppl 7A):27S-43. 
4 Sullivan PS, Hanson DL, Chu SY, et al. Epidemiology of anemia in human immunodeficiency virus (HIV)-infected persons: results from the multistate adult and adolescent spectrum of HIV disease surveillance project. Blood 1998;91:301-8.

5 Mocroft A, Kirk O, Barton SE, et al. Anaemia is an independent predictive marker for clinical prognosis in HIV-infected patients from across Europe. EuroSIDA Study Group. AIDS 1999;13:943-50.

6 Moore RD, Keruly JC, Chaisson RE. Anemia and survival in HIV infection. J Acquir Immune Defic Syndr Hum Retrovirol 1998;19:29-33.

7 Graham NM, Piantadosi S, Park LP, et al. CD4+ lymphocyte response to zidovudine as a predictor of AIDS-free time and survival time. $J$ Acquir Immune Defic Syndr 1993;6:1258-66.

8 Mocroft A, Lifson AR, Touloumi G, et al. Haemoglobin and anaemia in the smart study. Antivir Ther 2011;16:329-37.

9 Harris RJ, Sterne JAC, Abgrall S, et al. Prognostic importance of anaemia in HIV type-1-infected patients starting antiretroviral therapy: collaborative analysis of prospective cohort studies. Antivir Ther 2008;13:959-67.

10 McArthur JC, Hoover DR, Bacellar H, et al. Dementia in AIDS patients: incidence and risk factors. Multicenter AIDS cohort study. Neurology 1993;43:2245-52.

11 Anude CJ, Eze E, Onyegbutulem HC, et al. Immuno-virologic outcomes and immuno-virologic discordance among adults alive and on anti-retroviral therapy at 12 months in Nigeria. BMC Infect Dis 2013;13:113.

12 Kowalska JD, Mocroft A, Blaxhult A, et al. Current hemoglobin levels are more predictive of disease progression than hemoglobin measured at baseline in patients receiving antiretroviral treatment for HIV type 1 infection. AIDS Res Hum Retroviruses 2007;23:1183-8.

13 Lundgren JD, Mocroft A, Gatell JM, et al. A clinically prognostic scoring system for patients receiving highly active antiretroviral therapy: results from the EuroSIDA study. J Infect Dis 2002;185:178-87.

14 Zauli G, Davis BR, Re MC, et al. Tat protein stimulates production of transforming growth factor-beta 1 by marrow macrophages: a potential mechanism for human immunodeficiency virus-1-induced hematopoietic suppression. Blood 1992;80:3036-43.

15 Moore RD, Forney D. Anemia in HIV-infected patients receiving highly active antiretroviral therapy. J Acquir Immune Defic Syndr 2002;29:54-7.

16 Kerkhoff AD, Wood R, Cobelens FG, et al. Resolution of anaemia in a cohort of HIV-infected patients with a high prevalence and incidence of tuberculosis receiving antiretroviral therapy in South Africa. BMC Infect Dis 2014;14:3860.
17 Volberding PA, Levine AM, Dieterich D, et al. Anemia in HIV infection: clinical impact and evidence-based management strategies. Clin Infect Dis 2004;38:1454-63.

18 Harrison SA. Management of anemia in patients receiving protease inhibitors. Gastroenterol Hepatol 2012;8:254-6.

19 Mullick CMD, Belew Y. Clinical Review Dolutegravir NDA 204790 SN 00, 2012.

20 Kitahata MM, Rodriguez B, Haubrich R, et al. Cohort profile: the centers for AIDS research network of integrated clinical systems. Int J Epidemiol 2008;37:948-55.

21 Division of AIDS. Table for grading the severity of adult and pediatric adverse events. Available: http://rcc.tech-res.com/safetyandpharmac ovigilance/ [Accessed 3 Jan 2014].

22 Levey AS, Stevens LA, Schmid CH, et al. A new equation to estimate glomerular filtration rate. Ann Intern Med 2009;150:604-12.

23 Fredericksen R, Crane PK, Tufano J, et al. Integrating a web-based, patient-administered assessment into primary care for HIV-infected adults. J AIDS HIV Res 2012;4:47-55.

24 Laird NM, Ware JH. Random-effects models for longitudinal data. Biometrics 1982;38:963-74.

25 Miettinen OS. The need for randomization in the study of intended effects. Stat Med 1983;2:267-71.

26 Crane HM, Nance RM, Delaney JAC, et al. A comparison of adherence Timeframes using missed dose items and their associations with viral load in routine clinical care: is longer better? AIDS Behav 2017;21:470-80.

27 Wolde HM LW, YMelaku YA, Girmay KH. Incidence and risk factors of anemia among HIV/AIDS patients taking anti-retroviral therapy at tertiary hospitals in Addis Ababa, Ethiopia: a retrospective cohort study. Journal of HIVIAIDS and Infectious Diseases 2013.

28 Levine AM, Berhane K, Masri-Lavine L, et al. Prevalence and correlates of anemia in a large cohort of HIV-infected women: women's Interagency HIV study. J Acquir Immune Defic Syndr 2001;26:28-35.

29 Zhou J, Jaquet A, Bissagnene E, et al. Short-Term risk of anaemia following initiation of combination antiretroviral treatment in HIVinfected patients in countries in sub-Saharan Africa, Asia-Pacific, and central and South America. J Int AIDS Soc 2012;15:5.

30 Panel on Antiretroviral Guidelines for Adults and Adolescents. Guidelines for the use of antiretroviral agents in adults and adolescents living with HIV. Department of health and human services. Available: http://www.aidsinfo.nih.gov/ContentFiles/ AdultandAdolescentGL.pdf [Accessed Oct 2018].

31 Nance RM, Delaney JAC, Simoni JM, et al. Hiv viral suppression trends over time among HIV-infected patients receiving care in the United States, 1997 to 2015: a cohort study. Ann Intern Med 2018;169:376-84. 